\title{
Salas de Reuniões Virtuais para Apoio ao Ensino Remoto em Tempos de Pandemia: Uma Análise Comparativa
}

\author{
Fabrício Silva $^{1}$, Emanuel Lima ${ }^{1}$, Kelisson Vieira ${ }^{1}$, Geisy Anny Venancio ${ }^{1}$, \\ Priscila Fernandes $^{1}$, Vitor Bremgartner ${ }^{1}$ e Luis Rivero ${ }^{2}$ \\ ${ }^{1}$ Instituto Federal de Educação, Ciências e Tecnologia e do Amazonas (IFAM) \\ Manaus, AM - Brasil \\ ${ }^{2}$ Programa de Pós-Graduação em Ciência da Computação (PPGCC), \\ Universidade Federal do Maranhão (UFMA) - São Luís, MA - Brasil \\ $\{2021005488,2021000211,202100973$, geisy.venancio, \\ priscila.fernandes, vitorbref\}@ifam.edu.br,luisrivero@nca.ufma.br
}

\begin{abstract}
Due to the need to use information and communication technologies in remote learning as a result of the COVID-19 pandemic, there was a demand for systems that emulated virtual classrooms. To support teaching institutions and education professionals in choosing these technologies, a survey of the characteristics of these systems was carried out, thus allowing the selection of the best ones in the context of supporting teaching-learning for a given context. In this paper, a comparative analysis between online videoconferencing platforms that can be used to support remote learning is presented. As a result, we obtained a set of essential characteristics for those who are looking for free software in order to filter systems in support of remote learning.
\end{abstract}

Resumo. Devido à necessidade de utilizar tecnologias de informação e comunicação no ensino remoto decorrente da pandemia da COVID-19, houve uma procura por sistemas que emulassem salas de aula virtuais. Para apoiar instituições de ensino e profissionais da educação na escolha destas tecnologias, foi realizado um levantamento de características destes sistemas, permitindo assim, selecionar os melhores no âmbito de apoio ao ensino-aprendizagem para um determinado contexto. Neste artigo, é apresentada uma análise comparativa entre plataformas de videoconferência online que possam ser utilizadas no apoio ao ensino remoto. Como resultado, obteve-se um conjunto de características indispensáveis para quem está à procura de software livre com a finalidade de filtrar os sistemas no apoio ao ensino remoto.

\section{Introdução}

O surgimento do e-Learning como um novo cenário de utilização das tecnologias na educação e formação tem sido acompanhado de alguma discussão em torno da amplitude do próprio conceito de e-learning [Gomes 2005]. Dessa maneira, é possível afirmar que a educação não está ligada apenas aos espaços presenciais, como explica Gomes (2005): "O conceito de e-learning pode também estar associado a uma complementaridade entre actividades presenciais e actividades a distância tendo por suporte os serviços e tecnologias disponíveis na Internet (ou outra rede)". A princípio, qualquer metodologia de ensino viável a um indivíduo, torna-se fundamental para seu desenvolvimento profissional e ético 
perante a sociedade, e ao se tratar diretamente à Educação, sistemas de softwares livres que disponibilizem recursos essenciais para a formação de um indivíduo, são tão cruciais quanto o que seria uma aula presencial conhecida [Hernandez 2017]. Esses sistemas de comunicação e interação entre ambos interlocutores são responsáveis por proporcionar funcionalidades para os mesmos, com desígnio de ampliar a mesma performance ao educador e aluno, bem como oportunizar dentre os mais diversificados sistemas possíveis, podendo o usuário selecionar em qual plataforma almeja realizar suas reuniões virtuais.

As mudanças no sistema educacional tiveram de ser realizadas rapidamente, de modo que, de um dia para o outro, os professores precisaram transpor conteúdos e adaptar suas aulas presenciais para plataformas on-line com o emprego das Tecnologias de Informação e Comunicação (TICs), sem preparação para isso ou com a preparação superficial, também em caráter emergencial [Paudel 2021]. Neste novo contexto, identificou-se a necessidade de fazer um levantamento de quais são as melhores plataformas de videoconferência para realizar as reuniões de maneira mais eficaz e eficiente.

Com o advento e expansão da educação a distância (EAD) e também dos modelos híbridos, muitas ferramentas relacionadas às novas tecnologias da informação e comunicação já vinham sendo utilizadas para facilitar e intensificar o processo de ensino-aprendizagem [Ch and Popuri 2013]. Plataformas com uma variada gama de recursos digitais sempre estão sendo apresentadas aos alunos na tentativa de tornar o aprendizado mais estimulante e atrativo, o que diante do atual contexto, acabou por ser a alternativa para que o processo educativo não fosse interrompido totalmente [de Oliveira Zilse et al. 2021].

Se intensificando massivamente em decorrência do começo da pandemia do vírus SARS-COV-2 no mundo, cujo isolamento fez com que os trabalhos, reuniões de negócios e aulas se tornassem remotas. Especificamente, situações como essas tornaram-se evidentes em todos os países, destacando o Brasil entre eles. O Brasil adotou restrições durante a pandemia, dentre elas, o fechamento das instituições de ensino presencial em todo país. E com o isolamento social estabelecido em todo território nacional, as escolas, universidades, centros de ensino e outras instituições tiveram de se adaptar aos recursos disponíveis. Ferramentas de ensino remoto foram selecionadas para ministrar no modelo e-learning, assegurando o processo de ensino-aprendizagem de alunos e professores. Assim, plataformas que pudessem realizar videoconferência tornaram-se de extrema importância no mundo educativo desde então.

Devido à grande ascensão do uso desses meios de comunicação, houve um crescente aumento na concorrência e uma maior disponibilidade, de tal maneira que permite o consumidor filtrar e escolher o que melhor se encaixa em seu perfil [Singh and Awasthi 2020]. Nesse aspecto, existe uma grande tendência de que trabalhos comecem a ser realizados de maneira virtual, como explicado por Bridi et al (2020). Segundo o autor, milhões de trabalhadores e trabalhadoras tiveram suas atividades laborais impactadas e precisaram se ajustarem a uma nova forma de trabalhar, a de exercer suas funções profissionais de forma remota, em home-office. Segundo o IBGE PNADCOVID19, em maio de 2020, eram 8.7 milhões de trabalhadores em atividade remota. Consequentemente, foi possível perceber cada vez mais um aumento na procura de meios de comunicação que possibilitam a realização de atividades que migraram para as plataformas online. A fim de se obter uma melhor relação com os usuários, existe a busca 
por plataformas eficientes, de baixo custo e com boa estabilidade.

Este artigo tem como principal objetivo fazer um levantamento sobre as plataformas que possam ser utilizadas para videoconferência por professores e alunos, identificando suas particularidades e seus pontos positivos e negativos. A realização deste estudo visa identificar qual é a melhor opção disponível, avaliando dentre os serviços comparados os que melhor se relacionam com as atividades realizadas pelo usuário.

O restante deste trabalho está dividido em seções que abordam o que é um ambiente virtual de videoconferência. A Seção 2 apresenta o uso do e-learning e a importância do estudo destas plataformas, tendo em vista os desafios apresentados e a escassez deste tipo de comparação para auxiliar o usuário a realizar a melhor escolha A Seção 3 apresenta a metodologia, descrevendo quais foram os sistemas identificados para o uso de comunicação e interação entre educadores e alunos. Já, na Seção 4 são apresentados os resultados da análise das plataformas abordadas e analisadas. Finalmente, a Seção 5 aborda as considerações finais e trabalhos futuros que possam ser desenvolvidos a partir da análise de características dos sistemas.

\section{Plataformas de Reuniões Virtuais para Aulas em Videoconferência}

A Educação pode ser vista como um processo de descoberta, exploração e de observação, além de eterna construção do conhecimento. Diante disso, as características específicas da Realidade Virtual podem transformá-la num poderoso instrumento a serviço de todos que buscam a evolução da educação [Braga 2001]. Com esse aspecto, o ensino-aprendizagem no formato e-learning, torna-se indispensável para a formação dos discentes e para a contribuição na metodologia de ensino dos educadores, destacando-se no período da pandemia da COVID-19, como já esclarecido na introdução. Logo, para melhor atender o público-alvo de aulas em videoconferência no contexto atual, o estudo de análise de características conduzido e analisado foi correspondente aos softwares atuais, para ajudar os usuários a filtrar quais são as melhores plataformas a serem usadas, selecionando cada uma em etapas a serem destinadas à educação ou que se assemelhavam aos requisitos mínimos para a ministração de aulas síncronas.

O acesso à informação e os meios de comunicação se restringiram ao mundo virtual de forma abrupta, e os impactos foram sentidos na educação, atualmente restrita ao ambiente virtual [Cardoso et al. 2020]. De acordo com Khatib et al. (2020): "uma das saídas para a manutenção das atividades escolares e para garantir o cumprimento dos anos letivos foi o uso das aulas virtuais, em especial, por meio de videoconferências". Desse modo, se faz necessário o uso das Tecnologias da Informação e Comunicação (TICs) por docentes e discentes, de tal forma que possa garantir a participação e interação entre seus usuários por meio dos sistemas de apoio ao ensino remoto. As plataformas de reuniões virtuais são ferramentas capazes de disponibilizar funcionalidades essenciais aos seus usuários por videoconferência, como [Chen et al. 2020]: o chat público, chat privado, bloqueio de funções de usuários, remoção de usuários manual, enquete, notas compartilhadas, gravação gratuita, múltiplos moderadores e entre outras funcionalidades. Algumas delas não foram criadas especificamente com essa finalidade, porém, não deixam de ser primordiais para o apoio ao ensino remoto. Apesar de todos os recursos que as mesmas possuem, há sempre novas atualizações em seus sistemas. A princípio, as empresas administradoras dessas plataformas estão sempre em busca de novos recursos e competindo 
entre melhores serviços para seus consumidores.

As ferramentas de videoconferência além de transmitirem imagem e áudio, geralmente, possuem outras funcionalidades, entre elas, o quadro branco, transferência de arquivos, bate-papo e gravação. O quadro branco compartilhado é uma área de desenho compartilhada. Permite que os participantes importem imagens gráficas, escrevam, façam esquemas, o que pode esclarecer melhor um assunto. A transferência de arquivos entre os participantes é muito comum em ferramentas de videoconferência. Alguns sistemas permitem que aplicações sejam compartilhadas, permitindo que os participantes utilizem uma aplicação executada no computador de outro participante [do Prado et al. 2016].

Para assegurar o desenvolvimento de seus interlocutores, grandes empresas optaram por estabelecer o home office como solução, o que possibilitou o aperfeiçoamento e novas atualizações dessas plataformas, com o auxílio das TICs [Von Gaudecker et al. 2020]. Assim, sendo possível que se segregassem a partir dessas plataformas desenvolvidas, softwares livres no apoio ao ensino remoto em videoconferência, que possibilitem em tempo real aulas, palestras e reuniões semelhantes ao ensino presencial conhecido, podendo assegurar uma formação digna e compatível com outros métodos. Capazes de promover aos usuários a troca de conhecimentos de maneira fácil e prática através da conversação, apresentação de slides, quadro branco, imagens e entre outros aspectos. Sempre com o objetivo de tornar as aulas algo mais atrativo e que possa ser proveitosa para todos ali presentes. De acordo com Garcia (2013): "entre os vários objetivos para o uso da VC como recurso didático, destacam-se aqueles em que os professores auxiliam e motivam os alunos para a tomada de decisão e a criação de novas práticas docentes, ampliando a motivação dos mesmos para a aprendizagem”.

Diante do exposto, faz se necessário também analisar qual o impacto destas tecnologias no ensino remoto, através de sistemas que permitam executar reuniões virtuais. A seguir, é explicado o processo de execução desta pesquisa, comparando ferramentas existentes para este fim.

\section{Metodologia}

A metodologia foi baseada conforme dois artigos usados como modelo. O primeiro foi o artigo Estudo comparativo de ambientes gamificados no auxílio à aprendizagem [Pereira et al. 2019] e o segundo sendo Recursos para avaliação da aprendizagem: estudo comparativo entre ambientes virtuais de aprendizagem [Maquiné 2020], que serviram como modelo para realizar as comparações conforme visto nos mesmos. O estudo foi dividido em duas partes, a primeira parte foi realizada pela leitura bibliográfica para se ter melhor compreensão a respeito do tema. A segunda parte foi conduzida através de uma metodologia comparativa, utilizando a técnica de análise de características [Kitchenham 1997] que teve como objetivo analisar os principais programas e seus recursos disponíveis.

Foi realizada uma pesquisa online tanto no Google quanto nas lojas de aplicativos mobile para identificação de plataformas relacionadas com a realização de reuniões virtuais. Para a seleção das plataformas, utilizou-se como critério a usabilidade, simplicidade e a existência de versão gratuita, além da necessidade de que as mesmas possuam os seguintes requisitos fundamentais: chat público, compartilhamento de tela, bloqueio de funções de usuário, remoção de usuários e que elas possuíssem uma versão aberta ao público. Outros critérios foram levados em consideração, como: gravação de tela, quadro 
branco interativo, chat privado, enquete, notas compartilhadas, múltiplos moderadores, aplicativos mobile, levantar a mão, lembrete e código de convite. Estes têm como objetivo mostrar quais possuem mais recursos para seus usuários.

Algumas plataformas foram descartadas, pois não possuíam versão gratuita ou pela obrigatoriedade de se possuir e-mail institucional, não se encaixando assim nos requisitos fundamentais preestabelecidos. Dois exemplos são o BlueJeans e Open Meeting que são aplicativos para videoconferências focados em reuniões profissionais. Entretanto, este é justamente o problema que não os favorecem para o desenvolvimento de uma aula, visto que a necessidade de uma conta, e-mail e número de trabalho são requisitos dispensáveis e não necessários para uma aula, se tornando inviáveis para uso. A seguir, são descritas as plataformas que foram selecionadas para assim se fazer as comparações, com base nos requisitos destacados:

I. Discord: Pertencente a Discord Inc. lançada no dia 13 de maio de 2015, se tornou extremamente popular entre os jogadores para a comunicação nas partidas online. Também teve uma readaptação para se tornar acessível às escolas e universidades. A plataforma gratuita está disponível em versão mobile e desktop e traz segurança para evitar a presença de invasores.

II. Lark: A plataforma fundada em 2016 possui a combinação de mensagens, agendamento gerenciados pelo usuário e documentos online de acesso colaborativo em uma única plataforma. Busca revolucionar a forma com que os membros da equipe interagem para aumentar a eficiência. Possui uma interface simples de fácil acesso.

III. Zoom: Meetings: É descrito como um aplicativo ou plataforma de reuniões virtuais simplificado, que pode ser acessado tanto pelos computadores quanto pelos celulares. O serviço criado pelo norte-americano Eric Yuan, engenheiro líder da Cisco Systems em colaboração com sua unidade de negócios WebEx, pertencente a Zoom Vídeo Communications e começou a ser ofertado em janeiro 2013. Em maio do mesmo ano, já possuía a marca de um milhão de participantes.

IV. Google Meet: É uma plataforma desenvolvida pelo Google, que surgiu a partir da separação do recurso de vídeo e chat do Hangouts, para facilitar as reuniões de trabalho, permitindo o acesso por computadores e dispositivos móveis. Surgiu oficialmente em março de 2017, se tornando uma das principais plataformas de apoio ao ensino durante a pandemia.

V. Microsoft Teams: Desenvolvido pela Microsoft no dia 02 novembro de 2016, a Microsoft Teams se integra ao pacote de serviços e aplicações do Office 365. É uma plataforma unificada de comunicação e colaboração que combina batepapo, videoconferências, armazenamento de arquivos (incluindo colaboração em arquivos) e integração de aplicativos no local de trabalho.

VI. Skype: É um software desenvolvido em 2003 por Janus Friis e Niklas Zennstrom. A ferramenta permite a realização de chamadas de voz e vídeo para todo mundo através de computadores ou dispositivos móveis, em 2005 foi vendida para eBay e desde maio de 2011 pertence à Microsoft. Se tornou uma das plataformas de vídeo mais tradicionais do mercado.

VII. Whereby: É um aplicativo de videoconferências lançado em 22 de maio de 2015, disponível para dispositivos web ou móveis, que oferece áudio e vídeo gratuito sem a necessidade de possuir cadastro para acessar. O whereby é classificado 
como $\mathrm{n}^{\mathrm{o}} 1$ em facilidade de uso no G2 Crowd e atualmente o aplicativo possui mais de um milhão de downloads.

VIII. Elos: É uma plataforma de comunicação de áudio e vídeo, que promove a interação entre os usuários totalmente online, sem necessidade de instalação de aplicativos. A plataforma apenas precisa do acesso à internet. Esta plataforma foi criada em 2018 pela empresa Mconf, que é referência no ramo de videoconferências.

IX. BigBlueButton: Foi desenvolvido pelo programa Technology Innovation Management (TIM) do Instituto para Empreendedorismo e Comercialização de Tecnologia da Carleton University em Ottawa, Canadá, em 2007. Possui uma excelente interface e usabilidade de seus recursos, permitindo um grande controle sobre os mesmos.

X. Jitsi Meet: Criado em 2008, o jitsi é uma plataforma open source de videoconferência que tem como objetivo unir seus usuários de forma simples e organizada. Seu diferencial é a sua criptografia de ponta-a-ponta é sua interface simples.

\section{Resultados e Discussão}

Após a metodologia e a listagem de todos os sistemas, serão apresentados os resultados comparativos das plataformas de videoconferência selecionadas. A Tabela 1 apresenta as principais funcionalidades observadas em cada plataforma, organizando-as em seções por possuírem os melhores requisitos a serem usados, tendo como critério a análise de 17 (dezessete) recursos fundamentais para os usuários que irão utilizar os sistemas, listandoo em sequência na Tabela 1 a seguir.

Todas as plataformas foram comparadas usando os mesmos requisitos. O Discord foi o que apresentou melhor desempenho. Ele apresenta capacidade para suportar um total de 25 pessoas com video aberto e 99 em sala de voz, sendo o mais indicado entre todas as plataformas, pois cumpriu todos os requisitos, apresentando funcionalidades extras, tais como: bots, que podem ser utilizados para organizar as salas, enviar avisos e entre outras coisas que o destacam em relação aos demais, buscando sempre reduzir a distância entre seus usuários através de suas reuniões, trazendo discente e docente para um mesmo ambiente, exaltando a importância do diálogo para a formulação de ideias, fazendo com que a aprendizagem seja prejudicada o mínimo possível.

Ao aplicar o método comparativo, notou-se que o Lark cumpre com maestria todos os requisitos apresentados, tanto essenciais quanto complementares. Ele é fortemente indicado para o apoio ao ensino, pois sua interface simples assim como no Discord, ajuda os professores no ensino ficando atrás do Discord apenas por não oferecer bots.

Por sua vez, o Zoom Meetings cumpriu os requisitos tendo destaque apenas o fato de não possuir notas compartilhadas e a falta de legendas. Como não há necessidade de uma conexão de internet rápida e de qualidade elevada, esta plataforma se destaca em relação às outras. Ao ser avaliado, o Zoom se apresentou como um recurso eficaz para medição remota [dos Santos Junior and da Silva Monteiro 2020].

No Google Meet notou-se uma grande facilidade de acesso, sendo assim indicado para o uso escolar. Possui grande parte dos requisitos pressupostos. Pode-se ressaltar a identificação de alguns pontos para serem analisados com atenção, sendo eles: (a) a necessidade do cadastro de e-mail institucional para ser realizada a gravação das reuniões, 
Tabela 1. Resultado do comparativo das plataformas testadas

\begin{tabular}{|c|c|c|c|c|c|c|c|c|c|c|}
\hline $\begin{array}{l}\text { Requisitos / } \\
\text { Plataformas }\end{array}$ & 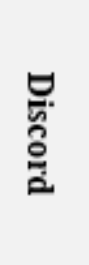 & 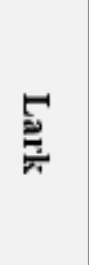 & $\begin{array}{l}\text { No } \\
\stackrel{0}{\mathrm{E}}\end{array}$ & 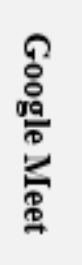 & 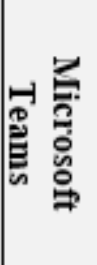 & 芯 & $\begin{array}{l}\sum_{1} \\
\stackrel{0}{0} \\
\stackrel{0}{0}\end{array}$ & 벟 & 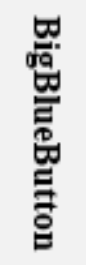 & 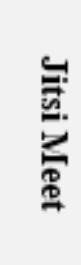 \\
\hline Versão gratuita & $\mathrm{Sim}$ & $\operatorname{Sim}$ & Sim & Sim & Sim & $\mathrm{Sim}$ & $\mathrm{Sim}$ & Sim & Sim & Sim \\
\hline Chat público & $\mathrm{Sim}$ & Sim & Sim & Sim & Sim & Sim & $\mathrm{Sim}$ & Sim & Sim & Sim \\
\hline $\begin{array}{l}\text { Compartilhamento de } \\
\text { tela }\end{array}$ & Sim & Sim & Sim & Sim & Sim & Sim & Sim & Sim & Sim & Sim \\
\hline $\begin{array}{l}\text { Bloqueio de funções de } \\
\text { usuário }\end{array}$ & Sim & Sim & Sim & Sim & Sim & Sim & Sim & Sim & Sim & Sim \\
\hline $\begin{array}{l}\text { Remoção de usuários } \\
\text { manual }\end{array}$ & Sim & Sim & Sim & Sim & Sim & Sim & Sim & Sim & Sim & Sim \\
\hline $\begin{array}{l}\text { Tempo de chamada } \\
\text { longo }\end{array}$ & Sim & Sim & Não & Não & Não & Sim & Não & Sim & Não & Sim \\
\hline Limite de pessoas & 250 & 350 & 100 & 100 & 100 & 100 & 100 & 15 & 100 & 100 \\
\hline Gravação g & Não & Sim & Sim & Não & Não & Sim & Não & Sim & Sim & Sim \\
\hline $\begin{array}{l}\text { Quadro branco } \\
\text { interativo }\end{array}$ & Não & Não & Sim & Não & Não & Sim & Sim & Sim & Sim & Não \\
\hline Chat privado & $\mathrm{m}$ & Sim & Sim & Nã & Sim & Sim & Não & Sim & Sim & Sim \\
\hline Enquete & Sim & Não & Sim & Não & Não & Sim & Não & Sim & Sim & Não \\
\hline Notas con & Sim & Sim & Sim & Não & Sim & Não & Não & Sim & Sim & Não \\
\hline Múltiplos moderadores & Sim & Sim & Sim & Não & Sim & Sim & Não & Sim & Sim & Não \\
\hline Aplicativo mobile & Sim & Sim & Sim & Sim & Sim & Sim & Sim & Não & Não & Sim \\
\hline Levantar a mão & Não & Sim & Sim & Sim & Sim & Sim & Sim & Sim & Sim & Sim \\
\hline Lembrete & Sim & Sim & Sim & Sim & Sim & Sim & Não & Sim & Não & Sim \\
\hline Código de convite & $\operatorname{Sim}$ & $\operatorname{Sim}$ & Sim & Sim & Sim & $\operatorname{Sim}$ & $\operatorname{Sim}$ & Sim & Sim & Sim \\
\hline
\end{tabular}

pois sem o mesmo a plataforma apresenta limite de tempo em sua gravação; (b) a ausência de alguns recursos como a incapacidade de realização de enquetes; e (c) inexistência de notas compartilhadas ou múltiplos moderadores.

No Microsoft Teams, percebeu-se a ausência de um quadro branco interativo, notas compartilhadas e recurso de gravação gratuita, sendo amenizado pela facilidade de acesso e interface simples. Sua vasta extensão possibilita a seus usuários que interliguem o Teams a produtos que não são da Microsoft. Ao utilizá-lo, o sistema proporcionará um ambiente digital aberto e que favorece a diversificada força de trabalho atualmente. Além disso, o sistema proporciona que estudantes, professores e funcionários se encontrem, trabalhem juntos, criem conteúdo e compartilhem recursos.

Quanto ao Skype, pode-se observar que é uma plataforma de fácil uso, porém foi insuficiente em alguns requisitos em comparação aos demais, como: falta de qua- 
dro branco interativo, chat privado e notas compartilhadas. No entanto, algumas dessas funções podem ser acessadas através de aplicativos terceiros, utilizando o compartilhamento de tela.

No comparativo, o Whereby, foi o que se mostrou mais limitação em relação aos requisitos aplicados. Por não possuir chat de conversas privado, notas compartilhadas, gravação gratuita, enquetes e limite de tempo de chamada, sendo indicada uma atualização para que possa assim satisfazer os seus usuários.

Destacamos um grande diferencial no Elos, que é a facilidade de comunicação com o suporte da plataforma, cumprindo com a maior parte dos requisitos propostos. O seu ponto negativo é o limite de pessoas na chamada, tendo a disponibilidade de 15 por chamada e apenas duas gravações de videoconferência na sua versão gratuita.

O BigBlueButton se destacou por possuir uma interface simples e de bom entendimento, em relação aos requisitos aplicados na comparação. Os pontos que foram insuficientes foram: o limite de tempo de 40 minutos por chamada e a falta de uma multiplataforma, dificultando assim o seu uso no apoio ao ensino.

O sistema jitsi apresentou um desempenho médio, pois priva o usuário de alguns recursos importantes. No entanto, possui chat privado e permite ao usuário o compartilhamento de vídeos do YouTube, um ponto importante a ser destacado.

É importante ressaltar que todas as plataformas possuem compartilhamento de tela que pode assim disponibilizar o uso de plataformas de aplicativos terceiros. Por exemplo, a lousa interativa do Google Meet é acessada dessa forma, fazendo com que alguns requisitos sejam cumpridos a partir desta opção. Porém, nesta análise, foi definido que os requisitos deveriam ser disponibilizados dentro da própria plataforma.

Após a realização das comparações constatou-se que todas as plataformas podem ser acessadas em grande parte dos países ao redor do mundo, sendo necessário apenas uma conexão à internet para seu funcionamento. Após a análise, notou-se o destaque das plataformas Discord e Lark, pois cumpriram todos os requisitos, sendo as que se apresentaram mais úteis para se lecionar, devido a facilidade de uso e manuseio de suas ferramentas. Ressalta-se as plataformas Zoom e Google Meet tendo cumprido quase todos os requisitos, podendo ser utilizado para o apoio ao ensino. Com esta análise, observase que existe uma maior necessidade de evolução dos softwares de videoconferência, principalmente em relação a realização de aulas online, fazendo com que as empresas modifiquem seus produtos para melhorar a performance e estabilidade da chamada no contexto educacional.

\section{Considerações Finais}

As plataformas de reuniões virtuais, quando direcionadas à Educação, possibilitam inúmeras funcionalidades no modelo e-learning de ensino aos alunos e professores, como destacado na seção anterior. A partir do estudo desenvolvido, observou-se que as plataformas de reuniões virtuais são ferramentas de extrema relevância no processo de ensinoaprendizagem. Estas ferramentas foram capazes de possibilitar o ensino básico e superior, além de ampliar as oportunidades de seus usuários a se conectarem uns com os outros, a qualquer hora e em qualquer lugar. Nesse contexto, indiscutivelmente, o desenvolvimento humano de cada indivíduo depende da sua capacidade de se adaptar a esses recursos e sis- 
temas. Além disso, qualquer pessoa que conheça o mínimo sobre elas tem a capacidade de manuseá-las no conforto de sua residência ou local de trabalho onde pretende realizar ou participar de reuniões online.

Neste trabalho de análise de características, foram selecionados os atributos de qualidade com o intuito de escolher as melhores plataformas para educadores e alunos. As instituições em todo o território nacional passaram a utilizar esses sistemas de comunicação para erguer e envolver a Educação no país com o objetivo de não deixar com que as aulas não fossem ministradas mesmo em situação de calamidade pública em meio a pandemia.

Este levantamento de características contribuiu para a formulação de pontos indispensáveis, tendo em vista que uma gama enorme de usuários é dependente destes meios de interação e comunicação no contexto de ensino. Vale ressaltar, que através de todos os requisitos abordados e analisados nessas plataformas, foi possível identificar quais são suas vantagens e desvantagens em relação às outras, e seus principais instrumentos de manuseio.

Espera-se que com o estudo realizado, as instituições sejam capazes de optar pela plataforma com mais funcionalidades disponíveis no formato e-learning de ensinoaprendizagem para seus discentes e docentes. Para que assim, possam sempre garantir que o conhecimento seja proveitoso, além de fornecer indícios às empresas donas desses serviços, mostrando quais funcionalidades necessitam de aperfeiçoamento e quais novos recursos podem ser desenvolvidos. Com isso, espera-se apoiar estas empresas, visando a melhor participação de seus consumidores nas plataformas por eles utilizadas.

Como trabalhos futuros, espera-se que novos estudos possam ser formulados contribuindo na identificação de requisitos, auxiliando na criação de novas ferramentas para o ensino remoto. Ao desenvolver sistemas de salas de reuniões virtuais, contribui-se para a aproximação do educador e do aluno, apoiando o ensino nos diversos níveis inicial, médio e superior.

\section{Agradecimentos}

Parte dos resultados apresentados neste trabalho foram obtidos através do PROJETO ARANOUÁ, financiado pela Samsung Eletrônica da Amazônia Ltda., com recursos previstos na Lei Federal n ${ }^{\circ} 8.387 / 1991$.

\section{Referências}

Braga, M. (2001). Realidade virtual e educação. Revista de biologia e ciências da terra, 1(1):0.

Bridi, M. A., Bohler, F. R., Zanoni, A. P., Braunert, M. B., Bernardo, K., Maia, F. L., FREIBERGER, Z. B., and GU, O. (2020). O trabalho remoto/home-office no contexto da pandemia covid-19. Curitiba: Universidade Federal do Paraná, Grupo de Estudos Trabalho e Sociedade.

Cardoso, C. A., Ferreira, V. A., and Barbosa, F. C. G. (2020). (des) igualdade de acesso à educação em tempos de pandemia: uma análise do acesso às tecnologias e das alternativas de ensino remoto. Revista Com Censo: Estudos Educacionais do Distrito Federal, 7(3):38-46. 
Ch, S. K. and Popuri, S. (2013). Impact of online education: A study on online learning platforms and edx. In 2013 IEEE International Conference in MOOC, Innovation and Technology in Education (MITE), pages 366-370. IEEE.

Chen, T., Peng, L., Yin, X., Rong, J., Yang, J., and Cong, G. (2020). Analysis of user satisfaction with online education platforms in china during the covid-19 pandemic. In Healthcare, volume 8, page 200. Multidisciplinary Digital Publishing Institute.

de Oliveira Zilse, S. S., Weber, A. L., and Floriani, J. R. (2021). Graduação em tempos de pandemia-aulas por videoconferência e percepção dos acadêmicos. Revista Paidéi@ Revista Científica de Educação a Distância, 13(23):1-19.

do Prado, M. d. F. W., Kich, S., Webber, C. G., et al. (2016). Avaliação de ferramentas de videoconferência para a plataforma moodle. RENOTE, 14(1).

dos Santos Junior, V. B. and da Silva Monteiro, J. C. (2020). Educação e covid-19: as tecnologias digitais mediando a aprendizagem em tempos de pandemia. Revista Encantar-Educação, Cultura e Sociedade, 2:01-15.

El Khatib, A. S. and Chizzotti, A. (2020). Aulas por videoconferência: uma solução para o distanciamento social provocado pela covid-19 ou um grande problema? uma solução para o distanciamento social provocado pela covid-19 ou um grande problema? Revista EDaPECI, 20(3):26-45.

Garcia, P. S., Malacarne, V., and de Tolentino Neto, L. C. B. (2013). O uso da videoconferência na educação: um estudo de caso com professores da educação básica. Reflexão e Ação, 21(2):10-33.

Gomes, M. J. (2005). E-learning: reflexões em torno do conceito.

Hernandez, R. M. (2017). Impact of ict on education: Challenges and perspectives. Journal of Educational Psychology-Propositos y Representaciones, 5(1):337-347.

Kitchenham, B. A. (1997). Evaluating software engineering methods and tools, part 7: planning feature analysis evaluation. ACM SIGSOFT software engineering Notes, 22(4):21-24.

Maquiné, G. O. (2020). Recursos para avaliação da aprendizagem: estudo comparativo entre ambientes virtuais de aprendizagem. In Anais do XXVI Workshop de Informática na Escola, pages 299-308. SBC.

Paudel, P. (2021). Online education: Benefits, challenges and strategies during and after covid-19 in higher education. International Journal on Studies in Education, 3(2):7085 .

Pereira, I. B., Santos, J., Suárez, P., and de Sousa, M. N. A. (2019). Estudo comparativo de ambientes gamificados no auxílio à aprendizagem. In Anais do Workshop de Informática na Escola, volume 25, pages 01-08.

Singh, R. and Awasthi, S. (2020). Updated comparative analysis on video conferencing platforms-zoom, google meet, microsoft teams, webex teams and gotomeetings. EasyChair: The World for Scientists, pages 1-9.

Von Gaudecker, H.-M., Holler, R., Janys, L., Siflinger, B., and Zimpelmann, C. (2020). Labour supply in the early stages of the covid-19 pandemic: Empirical evidence on hours, home office, and expectations. 